
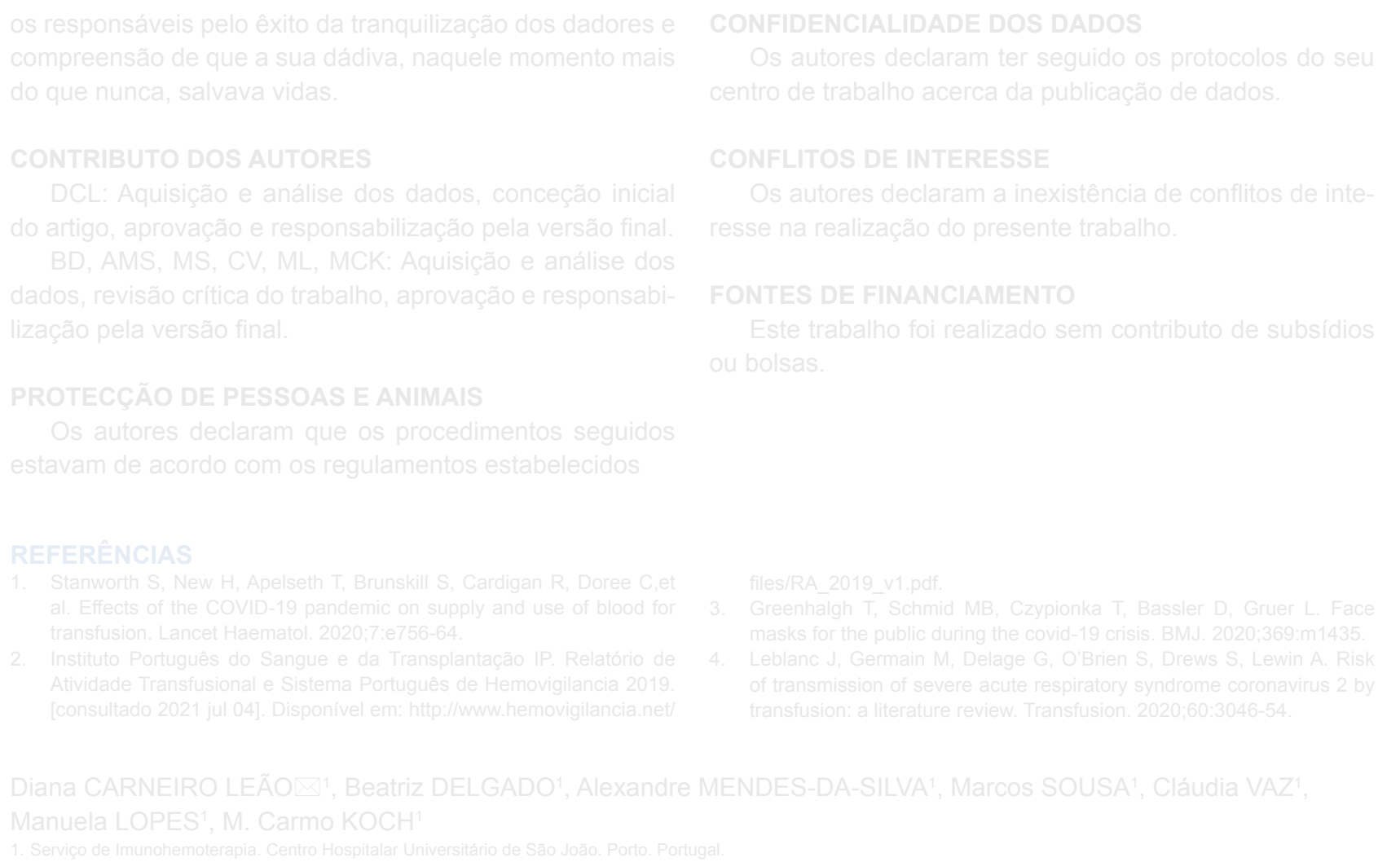

\section{Profilaxia do Tromboembolismo Venoso nos Doentes Psiquiátricos Internados}

\section{Prophylaxis of Venous Thromboembolism in Psychiatric Inpatients}

Palavras-chave: Embolia Pulmonar; Prevenção Primária; Tromboembolismo Venoso; Unidade Hospitalar de Psiquiatria Keywords: Primary Prevention; Pulmonary Embolism; Psychiatric Department Hospital; Venous Thromboembolism

Caro editor,

Em 2016 foi publicado nesta revista o artigo "Causas de Morte numa Unidade de Internamento de Agudos de Psiquiatria de um Hospital Geral Português", que concluiu que o tromboembolismo pulmonar foi a principal causa de morte no internamento psiquiátrico do Hospital Fernando Fonseca no período analisado de 1998 a $2013 .^{1}$

Este estudo alertou-nos para a problemática do tromboembolismo venoso (TEV) nos doentes psiquiátricos internados, sobre a qual gostaríamos de tecer algumas considerações.

De facto, estes resultados vêm de encontro com a evidência existente de que o TEV, nas suas principais formas de apresentação: trombose venosa profunda (TVP) e o tromboembolismo pulmonar (TEP), constitui uma das complicações mais frequentes no internamento hospitalar. ${ }^{2}$

Simultaneamente, apesar de os pacientes psiquiátricos internados manterem geralmente bons níveis de mobilidade, esta pode ser facilmente condicionada, dado que os pacientes apresentam frequentemente fatores de risco que os tornam particularmente vulneráveis à ocorrência do TEV, tais como a idade avançada, elevada prevalência de comorbilidades médicas, necessidade frequente de sedação ou de recurso a contenção física, catatonia, uso de antipsicóticos, dificuldades de comunicação e/ou atribuição comum dos sintomas físicos a causas psicossomáticas..$^{3,4}$

No entanto, a tromboprofilaxia farmacológica (uso de anticoagulantes como a enoxaparina) e/ou mecânica (ex. meias elásticas compressivas), abordagem comprovadamente segura e custo-efetiva, nem sempre é efetuada de forma adequada nos internamentos psiquiátricos, com prejuízos para os doentes e hospitais. ${ }^{3}$

Atentos à pertinência desta problemática, os autores das guidelines do National Institute for Health and Care Excellence (NICE), publicadas em 2018, incluem uma secção específica para os doentes psiquiátricos internados e 
recomendam que todos sejam submetidos a uma avaliação de risco para o TEV na admissão e ao longo do internamento sempre que a sua situação clínica se alterar. $^{5}$

Menos clara é a forma como esta avaliação de risco deve ser realizada., ${ }^{3,4}$ De facto, alguns autores têm tentado desenvolver ferramentas de avaliação de risco adaptadas para este subgrupo de doentes. ${ }^{4}$ Contudo, ainda não existe nenhuma que esteja formalmente validada nesta população, pelo que destacamos a necessidade de seguir as ferramentas de avaliação de risco e as diretrizes médicas gerais atualmente disponíveis, tais como as do NICE, ${ }^{5}$ tendo em consideração as suas potenciais limitações para que possam ser previstas e devidamente acauteladas. ${ }^{3,4}$

Perante o exposto, alertamos para necessidade de os serviços de psiquiatria desenvolverem e implementarem programas de tromboprofilaxia locais, com vista a garantir

\section{REFERÊNCIAS}

1. Barbosa S, Sequeira M, Castro S, Manso R, Klut Câmara C, Trancas $B$, et al. Causes of death in an acute psychiatric inpatient unit of a Portuguese general hospital. Acta Med Port. 2016;29:468-75.

2. Konstantinides S, Meyer G, Becattini C, Bueno H, Geersing G, Harjola V, et al. 2019 ESC Guidelines for the diagnosis and management of acute pulmonary embolism developed in collaboration with the European Respiratory Society (ERS): The Task Force for the diagnosis and management of acute pulmonary embolism of the European Society of Cardiology (ESC). Eur Respir J. 2019;54:1901647.

3. Wilkowska A, Kujawska-Danecka H, Hajduk A. Risk and prophylaxis of a adequada prevenção do TEV nos doentes psiquiátricos internados e, consequentemente, aumentar a sua segurança.

\section{CONTRIBUTO DOS AUTORES}

Ambos os autores contribuiram de igual forma para a conceção e desenho da pesquisa, redação do manuscrito e sua revisão crítica.

\section{CONFLITOS DE INTERESSE}

Os autores declaram não possuir quaisquer conflitos de interesse.

\section{FONTES DE FINANCIAMENTO}

O presente trabalho não foi suportado por nenhum subsídio ou bolsa.

venous thromboembolism in hospitalized psychiatric patients. A review. Psychiatr Pol. 2018;52:421-35.

4. Ishida T, Shibahashi K, Sugai S, Abe D, Hamabe $Y$, Kashiyama T, et al. Development of a risk stratification scoring system for deep vein thrombosis upon psychiatric admission. J Psychosom Res. 2021;147:110540.

5. National Guideline Centre. Venous thromboembolism in over 16s: Reducing the risk of hospital-acquired deep vein thrombosis or pulmonary embolism. London: National Institute for Health and Care Excellence (UK); 2018.

\section{Sónia PEREIRA $\rrbracket^{1}$, João SIMAS ${ }^{1}$}

1. Departamento de Psiquiatria e Saúde Mental. Centro Hospitalar do Tâmega e Sousa. Penafiel. Portugal.

Autor correspondente: Sónia Pereira.74416@chts.min-saude.pt

Recebido: 01 de agosto de 2021 - Aceite: 02 de agosto de 2021 - Online issue published: 01 de outubro de 2021

Copyright $\odot$ Ordem dos Médicos 2021

https://doi.org/10.20344/amp.16944 